\title{
Emociones Poderosas y no Poderosas ante Conflictos de Pareja: Diferencias de Género
}

\section{Powerful and Powerless Emotions in Partner Conflicts: Gender Differences}

\author{
Inmaculada Valor-Segura, Francisca Expósito y Miguel Moya \\ Universidad de Granada
}

\begin{abstract}
Resumen. Los conflictos son inherentes a cualquier tipo de relación interpersonal, y en el caso de las relaciones de pareja pueden tener innumerables efectos destructivos dentro de ésta. Las emociones adquieren una gran importancia en el estudio de los conflictos interpersonales (de pareja) ya que su expresión es natural en cualquier situación de conflicto. Se ha sugerido que las mujeres expresan "emociones no poderosas" como culpa, tristeza o miedo y los hombres, por el contrario, expresan en mayor medida "emociones poderosas" como furia o desprecio. El objetivo de este estudio consistió en investigar el tipo de emociones interpersonales que hombres y mujeres experimentaban ante distintas situaciones conflictivas de pareja. A su vez, se pretendía analizar el efecto de las emociones en la frecuencia de los conflictos. Se presentaron cinco situaciones hipotéticas conflictivas a un total de 142 estudiantes universitarios y se evaluó la intensidad de las emociones que sentían ante cada situación así como la frecuencia de los conflictos de pareja. Los resultados muestran diferencias de género en las emociones en cada situación conflictiva, de modo que las mujeres experimentaron con mayor intensidad todo tipo de emociones. Sin embargo, en hombres, las emociones poderosas predijeron una mayor frecuencia de conflictos en la pareja.

Palabras clave: emociones, conflictos de pareja, violencia de género.
\end{abstract}

\begin{abstract}
Conflict is inherent in all types of interpersonal relationships. It has especially important consequences in relationships involving high levels of interdependence, such as intimate relationships. Emotions are important to understand how people behave in their interpersonal relationships. Results from other studies suggest that women express powerless emotions like guilt, sadness or fear, and men express powerful emotions like anger or contempt. The aim of this study was to investigate the pattern of emotions that men and women feel when in conflictive situations with their partner. In addition, we examined the effect of emotions on the prevalence of partner conflicts. A total of 142 undergraduate students participated in our study. We used a mixed factorial design with 5 different types of interpersonal conflicts as a withinparticipants variable, and sex as a between-participants variable. Participants then rated the emotions felt in each conflictive situation, as well as the frequency of partner conflicts. Results showed sex differences in emotions in each conflictive situation. Women felt all emotions more intensely. In men, however, powerful emotions predicted a higher prevalence of partner conflicts.

Key words: emotions, partner conflicts, gender violence.
\end{abstract}

Las emociones son cruciales para entender como los individuos se comportan dentro de las relaciones interpersonales. Las relaciones íntimas representan uno de los principales contextos tanto para experimentar como para expresar emociones (Andersen y Guerrero, 1998; Berscheid, 1983; Laurenceau, Troy y Carver, 2005). Las emociones están fuertemente vinculadas a la desigualdad tradicional entre hombres y mujeres. En culturas occidentales como la nuestra, la creencia de la mujer como emocional y el hombre racional está ampliamente documentada (Fiske, Cuddy, Glick y Xu, 2002). Las mujeres han sido descritas con una mayor capacidad de empatía y sensibilidad hacia los senti-

La Correspondencia sobre este artículo debe enviarse a la primera autora al Departamento de Psicología Social, Facultad de Psicología. Universidad de Granada. Campus de Cartuja, s/n. 18071. Granada (España). E-mail: ivalor@ugr.es mientos de otros, mientras que los hombres se asocian con el control e inhibición de sus emociones (Brebner, 2003; Niedenthal, Krauth-Gruber y Ric, 2006).

En relación a la intensidad y la frecuencia de las emociones, numerosos estudios han mostrado que las mujeres sienten con mayor intensidad y más frecuentemente tanto emociones positivas (amor, afecto y alegría) como emociones negativas tales como tristeza, miedo, ira, angustia vergüenza y culpa (Brebner, 2003; Fishcher y Manstead, 2000). A su vez, numerosas investigaciones han sugerido que las mujeres expresan

Nota de Autor: Este trabajo ha sido posible gracias a la financiación concedida por la Junta de Andalucía al proyecto de excelencia "Violencia de género: análisis psicosocial de sus causas y estrategias para su reducción" (Ref. P06-HUM-01437), al proyecto de I+D SEJ2007-65816/PSIC del Ministerio de Educación y Ciencia, y al proyecto I+D+I 064/07 del Instituto de la Mujer. 
generalmente emociones que indican sumisión (docilidad), a menudo denominadas "emociones no poderosas" tales como tristeza, miedo, vergüenza o culpa. Los hombres, por el contrario, pese a ser considerados de manera general como menos emocionales que las mujeres, se les atribuye en mayor medida emociones que indican dominancia, a menudo denominadas "emociones poderosas" tales como ira, desprecio, disgusto y orgullo (Niedenthal et al., 2006; Timmers, Fischer y Manstead, 2003). Niedenthal et al., (2006) mostraron que en situaciones hipotéticas en las que inducían diferentes emociones, las mujeres manifestaban sentir con mayor intensidad miedo, tristeza, desilusión y furia en comparación con los hombres. En la misma línea, Brody, Lovas y Hay (1995) encontraron que las mujeres describen tener más miedo en situaciones hipotéticas temibles o atemorizantes y en aquellas situaciones que conciernen al comportamiento agresivo y hostil de un hombre (e.g., "él se está poniendo furioso y te está arrojando objetos").

Las emociones tienen una importante función social siendo inherentes al conflicto interpersonal y determinantes en la negociación (Davidson y Greenhalgh, 1999). Una emoción puede influir no sólo nuestro comportamiento, también en el comportamiento de otros y son cruciales para el entendimiento de cómo los individuos se comportan dentro de las situaciones estresantes o conflictivas. Los conflictos son inherentes a cualquier tipo de relación interpersonal, y en el caso de las relaciones de pareja pueden tener innumerables efectos destructivos dentro de ésta (Booth, Crouter y Clements, 2001). Un incremento de conflictos en la pareja y su inadecuada resolución, influye negativamente en la satisfacción con la relación y puede desembocar en graves infracciones incluyendo abuso emocional y físico (Holmes y Murray, 1996). Algunas emociones se han asociado con el incremento del riesgo de violencia en la pareja. Berkowitz (1993, 2000 ) ha sugerido que el afecto negativo (mal humor, desprecio, irritabilidad, ansiedad o ira) puede servir como motivador del conflicto y la agresión. En la presente investigación, se le presta una especial atención a la ira debido a la evidencia empírica que muestra la relación de esta emoción con el conflicto y la violencia dentro de las relaciones de pareja (Ellis y Malamuth, 2000; Marcus y Swett, 2003). Estudios clásicos sobre relación entre la ira y agresión han mostrado que el impulso o deseo de agresión estaba presente en el $40 \%$ de episodios conflictivos que cursaban con ira (Averill, 1983). Existen numerosos estudios que evidencian una menor aceptación de la mujer cuando exhibe conductas agresivas, de ahí que sea más probable que las mujeres encubran o contengan su ira, mientras que por el contrario, hay una mayor probabilidad de que los hombres la manifiesten o expresen (Etxebarria, Ortiz, Conejero y Pascual, 2009; Rivers, Brackett, Katulak y Salovey, 2007; Timmers, Fischer y Manstead, 1998. En definitiva, las características adscritas al rol feme- nino están relacionadas con la supresión de la ira, mientras que el rol masculino está asociado con su expresión externa (Kopper y Epperson, 1991, 1996).

El objetivo de este estudio consistió en investigar en primer lugar, el efecto del género en el tipo de emociones que experimentaban los participantes ante distintas situaciones conflictivas relacionadas con su relación de pareja. En concreto, se pretende analizar si las mujeres experimentan con mayor intensidad diferentes tipos de emociones en comparación con los hombres y, si esta tendencia aparece en todas las emociones o está limitada a las emociones estereotípicas de género. El segundo objetivo consistió en analizar el efecto de las "emociones poderosas" (furia, desprecio y enfado) en la frecuencia de los conflictos que los participantes tienen en su relación de pareja. Se espera que las "emociones poderosas" predigan una mayor frecuencia de conflictos, pero en hombres y no en mujeres.

\section{Método}

\section{Participantes y Diseño}

Participaron un total de 142 (75 mujeres y 67 hombres) estudiantes de la Universidad de Granada. Se utilizó un diseño factorial mixto con el tipo de conflicto interpersonal como variable intrasujeto (fueron presentados 5 situaciones conflictivas diferentes) y el género del participante como variable entre grupos.

\section{Procedimiento}

La muestra fue recogida mediante un muestreo incidental llevado a cabo en la ciudad de Granada. Dos evaluadores previamente entrenados solicitaban a los participantes su colaboración en el estudio informándoles del anonimato de sus respuestas y garantizándoles confidencialidad en el tratamiento de los datos.

\section{Instrumentos}

- Características sociodemográficas: sexo, edad, situación sentimental, tiempo en la relación, etc.

- Situaciones Conflictivas de Pareja. A los participantes se les presentaba 5 situaciones hipotéticas de conflicto con su pareja, basadas en The Proximal Antecedents of Violent Episodes Scale (PAVE) (Babcock, Costa, Green y Eckhardt, 2004). Estas situaciones trataban sobre violencia psicológica o física: (Situación 1) "Si mi pareja hace algo para ofenderme of faltarme el respeto"; (situación 2), "Si mi pareja me intimida físicamente durante una discusión; (situación 3), "Si mi 
pareja me ridiculiza o se burla de mî"; (situación 4), "Si mi pareja me levanta la voz de manera repetitiva" y (situación 5), "Si mi pareja manipula una discusión para llevar la razón".

- Emociones tras la situación conflictiva. Se les pedía a los participantes que evaluaran los diferentes tipos de emociones que sentían ante cada situación conflictiva (me sentiría culpable, enfadado/a, avergonzado/a, triste, indiferente, con desprecio, decepcionado/a, desilusionado/a, sentiría miedo y furioso/a). El formato de respuesta es tipo Likert de 6 puntos, donde 1 indicaba totalmente en desacuerdo y 6 totalmente de acuerdo.

- Escala de Conflictos para evaluar la frecuencia de los conflictos con su pareja (Tangney, 2000). Se compone de una escala de 15 ítems con un formato de respuesta tipo likert de 5 puntos en la que 1 indicaba "Esto nunca ocurre o ha ocurrido en mi relación" y 5 "Esto ocurre/ha ocurrido con mucha frecuencia en mi relación". Algunos ejemplos de ítems son: "Te hace comentarios negativos sobre alguien de tu familia", "habéis quedado para salir y en el último momento decide salir con sus amigos/as" o "se comporta de forma inapropiada durante la comida". El coeficiente alpha de escala total fue .82 .

\section{Resultados}

En general, los resultados mostraron diferencias de género en las emociones ante cada situación conflictiva. Tal y como puede observarse en la Tabla 1, en general, las mujeres expresaron con mayor intensidad todo tipo de emociones en comparación con los hombres.

En la situación 1 ("si mi pareja hace algo para ofenderme o faltarme el respeto") las mujeres indicaron sentir más tristeza $\mathrm{F}(1,136)=7,47, p=0,007$, más furia $F(1,136)=23,05, p<0,001$, enfado $\mathrm{F}(1,137)=$ $12,24, p=0,001$ y marginalmente mayor decepción $\mathrm{F}(1,137)=3,46, p=0,065$ y desilusión $\mathrm{F}(1,137)=$ $3,54, p=0,062$ que los hombres. A su vez, también se obtuvo efecto del género en la situación 2 ("Si mi pareja me intimida físicamente durante una discusión”), de modo que las mujeres sentían mayor decepción $\mathrm{F}(1,136)=7,16, p=0,008$, tristeza $\mathrm{F}(1,136)=10,61, p$ $=0,001$, furia $F(1,135)=23,33, p<0,001$, miedo $F(1,136)=33,06, p<0,001$, desilusión $F(1,136)=$ $19,04, p<0,001$, desprecio $\mathrm{F}(1,136)=6,44, p=0,012$ y mayor enfado $F(1,136)=23,38, p<0,001$ en comparación con los hombres. En la tercera situación (“ $S i$ mi pareja me ridiculiza o se burla de mí"), las mujeres experimentaron más decepción $F(1,136)=5,50, p=$ 0,020 , tristeza $F(1,135)=11,60, p=0,001$, furia $F(1,136)=7,22, p=0,008$, desilusión $F(1,136)=$ $16,13, p<0,001$ y mayor enfado $F(1,136)=10,30, p=$ 0,002 que los hombres. En la situación 4 ( "Si mi pare- ja me levanta la voz de manera repetitiva”) se observó que las mujeres sintieron con mayor intensidad, tristeza $F(1,135)=5,92, p=0,016$, furia $F(1,134)=6,91, p$ $=0,010$, miedo $F(1,134)=7,20, p=0,008$, desilusión $F(1,134)=5,89, p=0,017$ y marginalmente mayor decepción $F(1,134)=3,30, p=0,068$, desprecio $F(1,135)=3,85, p=0,052$ y enfado $F(1,136)=3,31, p$ $=0,071$ que los hombres. Sin embargo en esta situación los hombres experimentaron más culpa $F(1,135)$ $=5,92, p=0,016$ que las mujeres. Por último, se obtuvo efecto del género en la situación 5 ("Si mi pareja manipula una discusión para llevar la razón"), de modo que las mujeres ante esta situación hipotética sintieron más tristeza $F(1,135)=4,50, p=0,036$, furia $F(1,135)=8,37, p=0,004$ y enfado $F(1,136)=4,18, p$ $=0,043$ que los hombres. Sin embargo, los hombres se sintieron más avergonzados si su pareja manipula la conversación para llevar la razón $F(1,135)=4,03, p=$ 0,047 .

\section{Efecto de las emociones poderosas y no poderosas en la frecuencia del conflicto}

Para poder determinar qué tipo de emociones (poderosas o no poderosas) podrían predecir la frecuencia de conflictos dentro de la pareja, en primer lugar se halló la puntuación media total de los participantes en las emociones poderosas (furia, desprecio y enfado) y la puntuación media total de las emociones no poderosas (culpa, vergüenza, tristeza y miedo). A continuación se realizó un análisis de regresión múltiple en el que las variables predictoras fueron la puntuación total de las emociones poderosas y las emociones no poderosas y como variable criterio la puntuación total en la frecuencia de los conflictos con la pareja. Los resultados mostraron, tal y como se puede observar en la Tabla 2, que las emociones poderosas predecían una mayor frecuencia de los conflictos dentro de la pareja en hombres $\left.\left(R^{2}=0,10\right), F(1,64)=3,41, p=0,039\right)$. La regresión para la muestra de mujeres no fue significativa $\left(R^{2}\right.$ $=0,02), F(1,72)=0,87, p=0,42)$.

\section{Discusión}

Los conflictos son inherentes en cualquier tipo de relación interpersonal. Las emociones tienen una importante función social y nos ayudan a relacionarnos con los demás, siendo inherentes a todo tipo de conflicto pero sobre todo, en aquellos que surgen en relaciones significativas para el ser humano como son las relaciones interpersonales íntimas (Davidson y Greenhalgh, 1999). Los resultados de la presente investigación han mostrado que en general, ante situaciones conflictivas en la pareja, las mujeres experimentaban con mayor intensidad que los hombres, todo tipo de 
Tabla 1. Diferencias de género en las emociones experimentadas ante las situaciones conflictivas

\begin{tabular}{|c|c|c|c|c|c|c|c|c|c|c|}
\hline & \multicolumn{10}{|c|}{ Violencia Psicológica / Física } \\
\hline & \multicolumn{2}{|c|}{ Situación 1} & \multicolumn{2}{|c|}{ Situación 2} & \multicolumn{2}{|c|}{ Situación 3} & \multicolumn{2}{|c|}{ Situación 4} & \multicolumn{2}{|c|}{ Situación 5} \\
\hline & Hombre & Mujer & Hombre & Mujer & Hombre & Mujer & Hombre & Mujer & Hombre & Mujer \\
\hline & $\begin{array}{c}\mathrm{M} \\
(\mathrm{Sd})\end{array}$ & $\begin{array}{c}\mathrm{M} \\
(\mathrm{Sd})\end{array}$ & $\begin{array}{c}\mathrm{M} \\
(\mathrm{Sd})\end{array}$ & $\begin{array}{c}\mathrm{M} \\
(\mathrm{Sd})\end{array}$ & $\begin{array}{c}\mathrm{M} \\
(\mathrm{Sd})\end{array}$ & $\begin{array}{c}\mathrm{M} \\
(\mathrm{Sd})\end{array}$ & $\begin{array}{c}\mathrm{M} \\
(\mathrm{Sd})\end{array}$ & $\begin{array}{c}\mathrm{M} \\
(\mathrm{Sd})\end{array}$ & $\begin{array}{c}\mathrm{M} \\
(\mathrm{Sd})\end{array}$ & $\begin{array}{c}\mathrm{M} \\
(\mathrm{Sd})\end{array}$ \\
\hline Culpable & $\begin{array}{c}1,75 \\
(1,37)\end{array}$ & $\begin{array}{c}1,68 \\
(1,24)\end{array}$ & $\begin{array}{c}1,66 \\
(1,29)\end{array}$ & $\begin{array}{c}1,45 \\
(1,24)\end{array}$ & $\begin{array}{c}1,75 \\
(1,38)\end{array}$ & $\begin{array}{c}1,47 \\
(1,12)\end{array}$ & $\begin{array}{c}1,70 \\
(1,27)\end{array}$ & $\begin{array}{c}1,29 \\
(0,68)\end{array}$ & $\begin{array}{c}1,47 \\
(0,89)\end{array}$ & $\begin{array}{c}1,40 \\
(1,15)\end{array}$ \\
\hline Avergonzado/a & $\begin{array}{c}2,92 \\
(1,74)\end{array}$ & $\begin{array}{c}2,74 \\
(1,72)\end{array}$ & $\begin{array}{c}2,61 \\
(1,85)\end{array}$ & $\begin{array}{c}2,67 \\
(2,04)\end{array}$ & $\begin{array}{c}3,59 \\
(1,86)\end{array}$ & $\begin{array}{c}3,84 \\
(1,99)\end{array}$ & $\begin{array}{c}2,84 \\
(1,87)\end{array}$ & $\begin{array}{c}2,90 \\
(2,06)\end{array}$ & $\begin{array}{c}2,56 \\
(1,86)\end{array}$ & $\begin{array}{c}1,97 \\
(1,58)\end{array}$ \\
\hline Decepcionado/a & $\begin{array}{c}4,52 \\
(1,69)\end{array}$ & $\begin{array}{c}5,01 \\
(1,48)\end{array}$ & $\begin{array}{c}4,03 \\
(1,90)\end{array}$ & $\begin{array}{c}4,88 \\
(1,79)\end{array}$ & $\begin{array}{c}4,46 \\
(1,79)\end{array}$ & $\begin{array}{c}5,11 \\
(1,46)\end{array}$ & $\begin{array}{c}3,89 \\
(1,78)\end{array}$ & $\begin{array}{c}4,47 \\
(1,89)\end{array}$ & $\begin{array}{c}3,74 \\
(1,89)\end{array}$ & $\begin{array}{c}3,78 \\
(2,02)\end{array}$ \\
\hline Triste & $\begin{array}{c}4,13 \\
(1,79)\end{array}$ & $\begin{array}{c}4,86 \\
(1,37)\end{array}$ & $\begin{array}{c}3,63 \\
(1,89)\end{array}$ & $\begin{array}{c}4,67 \\
(1,86)\end{array}$ & $\begin{array}{c}3,90 \\
(1,85)\end{array}$ & $\begin{array}{c}4,92 \\
(1,62)\end{array}$ & $\begin{array}{c}3,17 \\
(1,98)\end{array}$ & $\begin{array}{c}4,34 \\
(1,95)\end{array}$ & $\begin{array}{c}2,73 \\
(1,70)\end{array}$ & $\begin{array}{c}3,40 \\
(1,93)\end{array}$ \\
\hline Furioso/a & $\begin{array}{c}3,52 \\
(1,78)\end{array}$ & $\begin{array}{c}4,85 \\
(1,47)\end{array}$ & $\begin{array}{c}3,70 \\
(1,81)\end{array}$ & $\begin{array}{c}5,14 \\
(1,66)\end{array}$ & $\begin{array}{c}3,73 \\
(1,85)\end{array}$ & $\begin{array}{c}4,59 \\
(1,89)\end{array}$ & $\begin{array}{c}3,59 \\
(1,86)\end{array}$ & $\begin{array}{c}4,44 \\
(1,91)\end{array}$ & $\begin{array}{c}3,55 \\
(1,69)\end{array}$ & $\begin{array}{c}4,42 \\
(1,84)\end{array}$ \\
\hline Despreciado/a & $\begin{array}{c}3,92 \\
(1,78)\end{array}$ & $\begin{array}{c}4,15 \\
(1,74)\end{array}$ & $\begin{array}{c}3,48 \\
(1,93)\end{array}$ & $\begin{array}{c}4,34 \\
(2,01)\end{array}$ & $\begin{array}{c}4,02 \\
(1,89)\end{array}$ & $\begin{array}{c}4,46 \\
(1,89)\end{array}$ & $\begin{array}{c}3,47 \\
(1,82)\end{array}$ & $\begin{array}{c}4,11 \\
(1,98)\end{array}$ & $\begin{array}{c}3,39 \\
(1,80)\end{array}$ & $\begin{array}{c}3,36 \\
(1,90)\end{array}$ \\
\hline Desilusionado/a & $\begin{array}{c}4,45 \\
(1,50)\end{array}$ & $\begin{array}{c}4,93 \\
(1,48)\end{array}$ & $\begin{array}{c}3,89 \\
(1,89)\end{array}$ & $\begin{array}{c}5,16 \\
(1,53)\end{array}$ & $\begin{array}{c}4,17 \\
(1,88)\end{array}$ & $\begin{array}{c}5,29 \\
(1,29)\end{array}$ & $\begin{array}{c}3,84 \\
(1,88)\end{array}$ & $\begin{array}{c}4,60 \\
(1,78)\end{array}$ & $\begin{array}{c}3,48 \\
(1,84)\end{array}$ & $\begin{array}{c}3,82 \\
(1,90)\end{array}$ \\
\hline Indiferente & $\begin{array}{c}2,55 \\
(1,57)\end{array}$ & $\begin{array}{c}2,14 \\
(1,62)\end{array}$ & $\begin{array}{c}2,15 \\
(1,55)\end{array}$ & $\begin{array}{c}2,49 \\
(2,03)\end{array}$ & $\begin{array}{c}2,30 \\
(1,57)\end{array}$ & $\begin{array}{c}1,93 \\
(1,66)\end{array}$ & $\begin{array}{c}2,32 \\
(1,54)\end{array}$ & $\begin{array}{c}2,14 \\
(1,74)\end{array}$ & $\begin{array}{c}2,37 \\
(1,57)\end{array}$ & $\begin{array}{c}2,30 \\
(1,80)\end{array}$ \\
\hline Enfadado/a & $\begin{array}{c}4,02 \\
(1,60)\end{array}$ & $\begin{array}{c}4,93 \\
(1,49)\end{array}$ & $\begin{array}{c}3,90 \\
(1,89)\end{array}$ & $\begin{array}{c}5,30 \\
(1,48)\end{array}$ & $\begin{array}{c}4,06 \\
(1,87)\end{array}$ & $\begin{array}{c}4,99 \\
(1,51)\end{array}$ & $\begin{array}{c}4,18 \\
(1,75)\end{array}$ & $\begin{array}{c}4,74 \\
(1,83)\end{array}$ & $\begin{array}{c}3,80 \\
(1,71)\end{array}$ & $\begin{array}{c}4,42 \\
(1,86)\end{array}$ \\
\hline
\end{tabular}

Nota: Situación 1: "Si mi pareja hace algo para ofenderme o faltarme el respeto'; situación 2: "Si mi pareja me intimida físicamente durante una discusión'; situación3: "Si mi pareja me ridiculiza o se burla de mí'; situación 4: "Si mi pareja me levanta la voz de manera repetitiva”, y situación 5: "Si mi pareja manipula una discusión para llevar la razón".

Tabla 2. Análisis de Regresión Lineal de las emociones poderosas y no poderosas como predictoras de la frecuencia de los conflictos en la pareja

\begin{tabular}{|c|c|c|c|c|c|c|}
\hline & \multicolumn{2}{|c|}{ Beta } & \multicolumn{2}{|c|}{$\mathrm{t}$} & \multicolumn{2}{|c|}{ Sig. } \\
\hline & Hombres & Mujeres & Hombres & Mujeres & Hombres & Mujeres \\
\hline Emociones poderosas & 0,38 & 0,15 & 2,40 & 1,03 & 0,019 & 0,31 \\
\hline Emociones no poderosas & $-0,12$ & 0,01 & $-0,77$ & 0,09 & 0,44 & 0,93 \\
\hline
\end{tabular}

emociones, de acuerdo con el estereotipo de "expresividad". A pesar de que a priori se podría esperar que los hombres expresaran más emociones dominantes o "poderosas" (furia, enfado y desprecio) y las mujeres emociones menos poderosas o de sumisión (culpa, tristeza, miedo,...), los resultados han mostrado que las mujeres han sentido con mayor intensidad tanto emociones "poderosas" como "no poderosas". En concreto, ante situaciones hipotéticas de violencia física o psicológica con la pareja, las mujeres describían sentir mas emociones negativas, especialmente en todas las situaciones sintieron más tristeza, así como en casi todas las situaciones conflictivas se sentían decepcionadas y desilusionadas si esas situaciones ocurrieran en su rela- ción de pareja. A su vez, las mujeres también sintieron más intensidad de furia y enfado ante las situaciones presentadas en comparación con los hombres. Este dato queda avalado por una amplia literatura que documenta la creencia estereotípica de la mujer como más expresiva y emocional (Brebner, 2003; Fishcher y Manstead, 2000; Fiske et al., 2002; Niedenthal et al., 2006; Timmers et al., 1998). De todas las situaciones, la situación que mayor impacto emocional ha tenido para las mujeres fue la situación 2 "si mi pareja me intimida físicamente durante una discusión". Ante esta situación las mujeres se sintieron más tristes, con miedo, decepcionadas, con desprecio, enfadadas y desilusionadas. Este resultado es congruente con las ex- 
pectativas que las mujeres tienen en su relación de pareja, en la que se espera encontrar apoyo emocional, respeto, compañerismo y lealtad, por lo que cualquier resultado diametralmente opuesto a estas expectativas, podría producir una gran decepción con la relación idealizada. Por otra parte, tan sólo en dos de las situaciones presentadas, los hombres obtuvieron puntuaciones más altas en la emoción de culpa ("si mi pareja me levanta la voz de manera repetitiva") y vergüenza ("si mi pareja manipula la conversación para llevar la razón”). Estos resultados, si bien necesitan ser analizados con mayor profundidad, podrían ponernos sobre la pista de la importancia que los hombres otorgan a llevar las riendas y tener el control en sus relaciones de pareja. De este modo, cualquier situación que cuestione este "control", produce en ellos emociones negativas que indican de alguna manera su incapacidad para hacerse valer en la relación.

Por último, un resultado interesante fue que a pesar de que las mujeres obtuvieron mayores puntuaciones en todo tipo de emociones, tan sólo en hombres las emociones poderosas predecían la frecuencia de los conflictos. Esto es, los participantes varones que se sintieron más furiosos, despreciados y enfadados ante situaciones conflictivas de pareja tenían más conflictos en su relación de pareja. En las mujeres las emociones de dominación o poderosas no predecían la prevalencia de los conflictos de pareja. En definitiva, los resultados obtenidos pueden sugerir que si bien las mujeres son capaces de experimentar más enfado o furia ante situaciones de conflicto en la pareja, esta mayor intensidad emocional no se traduce en una mayor conflictividad en su relación de pareja. Este resultado puede ser debido a que las características adscritas al rol femenino y que han sido ampliamente interiorizadas por las propias mujeres, están asociadas a reconducir o controlar la ira, mientras que el rol masculino facilita que los hombres puedan expresar la ira de manera externa y explícitamente (Etxebarria et al., 2009; Kopper y Epperson, 1991, 1996; Timmers et al., 1998; Rivers et al., 2007), lo que se traduce, entre otras consecuencias, en un incremento en la frecuencia de conflictos.

De alguna manera, mediante el proceso de socialización se asumen roles y normas de comportamiento asociados al género, generándose expectativas diferentes respecto a lo que resulta "adecuado" para hombres y para mujeres en el seno de la relación de pareja. El contexto sociocultural y los roles de género asignados a los hombres y a las mujeres podrían estar desempeñando un papel fundamental en la forma en la que se generan expectativas diferentes para hombres y mujeres respecto a cuál debe ser su rol en la relación y ante el conflicto. De la misma manera que la sociedad establece unas serie de normas respecto a cómo deben conformarse las relaciones entre hombres y mujeres, también establece normas respecto a cómo ambos deben responder ante situaciones de conflicto.

\section{Referencias}

Andersen, P. A., y Guerrero, L. K. (1998). Principles of communication and emotion in social interaction. En P. A. Andersen y L. K. Guerrero (Eds.), Communication and emotion: Research, theory, applications, and contexts (pp. 49-96). San Diego, CA: Academic Press.

Averill, J. R. (1983). Studies on anger and aggression: Implications for theories of emotion. American Psychologist, 38, 1145-1160.

Babcock, J. C., Costa, D. M., Green, C. E. y Eckhardt, C. I. (2004). What situations induce intimate partner violence? A reliability and validity study of the proximal antecedents to violent episodes (PAVE) Scale. Journal of Family Psychology, 18, 433-442.

Berkowitz, L. (1993). Aggression: its causes, consequences, and control. New York: McGraw-Hill.

Berkowitz, L. (2000). Causes and consequences of feelings. Cambridge, UK: Cambridge.

Berscheid, E. (1983). Emotion. En H. H. Kelley, E. Berscheid, A. Christensen, J.H. Harvey, T. L. Huston, G. Levinger, et al. (Eds.), Close relationships (pp. 110-168). New York: Freeman.

Booth, A., Crouter, A. C. y Clements, M. (2001). Couples in conflict. Mahwah, NJ: Lawrence Erlbaum.

Brody, L. R., Lovas, G. S. y Hay, D. H. (1995). Gender differences in anger and fear as a function of situational context. Sex Roles, 32, 47-79.

Davidson, M. N. y Greenhalgh, L. (1999). The role of emotion in negotiation: The impact of anger. En R. J. Bies, R. J. Lewicki y B. H. Sheppard, (Eds.), Research on Negotiation in Organizations (pp. 3-26). Greenwich, CT: JAI Press Inc.

Etxebarria, I., Ortiz, M. J., Conejero, S. y Pascual, A. (2009). Intensity of habitual guilt in men and women: Differences in interpersonal sensitivity and the tendency towards anxious-aggressive guilt. The Spanish Journal of Psychology, $12,540-554$.

Ellis, B. J. y Malamuth, N. M. (2000). Love and anger in romantic relationships: a discrete systems model. Journal of Personality, 68, 525-556.

Fischer, A. H. y Manstead, S. R. (2000). The relation between gender and emotions in different cultures. En A. H. Fischer (Ed.), Gender and emotion: Social psychological perspectives (pp. 71-94). Cambridge: Cambridge University Press.

Fiske, S. T., Cuddy, A. J. C., Glick, P. y Xu, J. (2002). A model of (often mixed) stereotype content: Competence and warmth respectively follow from status and competition. Journal of Personality and Social Psychology, 82, 878-902.

Holmes, J. G. y Murray, S. L. (1996). Interpersonal Conflict. En E. T. Higgins y A. Kruglanski (Eds.), Social Psychology: Handbook of basic mechanisms and processes (pp. 622-654). New York: Guilford.

Kopper, B. A. y Epperson, D. L. (1991). Women and anger. Sex and sex-role comparisons in the expression of anger. Psychology of Women Quarterly, 15, 7-14. 
Kopper, B. A. y Epperson, D. L. (1996). The experience and expression of anger: Relationships with gender, genderrole socialisation, depression, and mental health functioning. Journal of Counselling Psychology, 43, 158-165.

Laurenceau, J. P., Troy, A. B. y Carver, C. S. (2005). Two distinct emotional experiences in romantic relationships: effects of perceptions regarding approach of intimacy and avoidance of conflict. Personality and Social Psychology Bulletin, 31, 1123-1133.

Marcus, R. F. y Swett, B. (2003). Violence in close relationships: The role of emotion. Aggresion and Violent Behavior, 8, 313-327.

Niedenthal, P., Krauth-Gruber, S. y Ric, F. C. (2006).
Psychology of Emotion. New York: Psychology Press.

Rivers, S. E. Brackett, M. A., Katulak, N. A. y Salovey, P. (2007). Regulating anger and sadness: an exploration of discrete emotions in emotion regulation. Journal of Happiness Studies, 8, 393-427.

Timmers, M., Fischer, A. H. y Manstead, A. S. R. (1998). Gender differences in motives for regulating emotions. Personality and Social Psychology Bulletin, 24, 974985.

Timmers, M., Fischer, A. H. y Manstead, A. S. R. (2003). Ability versus vulnerability: Beliefs about men's and women's emotional behaviour. Cognition and Emotion, 17, 41-63.

Manuscrito Recibido: 03/03/2010

Revisión Recibida: 20/05/2010

Manuscrito Aceptado: 20/05/2010 